\title{
An easy and general method for quantifying Azithromycin dihydrate in a matrix of amorphous Azithromycin
}

\author{
Jose Montejo-Bernardo, ${ }^{\text {a }}$ Santiago García-Granda, ${ }^{* a}$ Miguel Bayod-Jasanada, \\ Isidro Llorente, ${ }^{b}$ and Luján Llavona ${ }^{b}$ \\ ${ }^{a}$ X-Ray Group, Department of Physical and Analytical Chemistry, University of Oviedo, 33006 \\ Oviedo, Asturias, Spain \\ ${ }^{b}$ Astur-Pharma S.A. Department of Research and Development, 33192 Silvota, Asturias, Spain \\ E-mail:sgg@fq.uniovi.es
}

\section{This paper is dedicated to Professors Jose Elguero and Pedro Molina on the happy occasion of their $70^{\text {th }}$ and $60^{\text {th }}$ birthdays}

(received 30 Dec 04; accepted 12 Apr 05; published on the web 14 Apr 05)

\begin{abstract}
Azithromycin is a macrolide antibiotic with several important advantages over the parent drug, erythromycin A, such as a wider spectrum of activity, better bioavailability and pharmacological and pharmacokinetic properties, which has a crystalline form and several patented pseudopolymorphs. There are also amorphous forms which show remarkable advantages over the known crystalline forms, but spontaneously produce the dihydrate under some particular conditions. The amount of crystalline phase is usually below $10 \%$ and typically $3-5 \%$. We report a new procedure for directly and easily quantifying the crystalline dihydrate form in samples of amorphous Azithromycin by using the X-ray diffraction pattern of the mixture and combining the most intense peak of the dihydrate and an estimation of the background, which gives a good linear relationship between the percentage of crystalline phase and the area of the main peak.
\end{abstract}

Keywords: Azithromycin, pseudopolymorphs, quantification, X-ray powder

\section{Introduction}

The efficient and accurate quantification of amorphous or crystalline phases in pharmaceutical samples is a challenging task in the modern pharmaceutical industry. The presence of an undesirable phase can mean important changes in the chemical and physical properties of the substance, affecting its biopharmaceutical features such as stability, solubility, or bio-availability (i.e., how metabolically available a drug or other chemical becomes to the target tissue after it is introduced into a person's body). 
The determination of the percentage of an amorphous phase in a crystalline sample has largely been studied in recent years in several materials such as drugs, ${ }^{1,2}$ cements, or ceramics, using X-ray powder diffraction, with the help of internal standards in most cases.

The quantification of crystalline phase/s in amorphous matrices is less studied, and has been restricted to drugs (to analyze the active pharmaceutical ingredient and the excipients). Nowadays, the X-ray powder pattern of the pure crystalline phase is either used as a reference when the percentage of crystalline phase in the amorphous matrix is high, ${ }^{3}$ or as an internal standard, ${ }^{4}$ where the Rietveld method is applied. In other applications, mainly for excipients, the percent weight of the crystalline phase in the sample is obtained by relating the integrated intensities of some diffraction peaks. ${ }^{2,5}$

This paper describes a method of quantification in a drug of low percentages of crystalline phase within an amorphous matrix by using X-ray powder diffraction patterns without using internal standards or the pattern of the pure crystalline phase. The primary subjects of our quantification are mixtures of the antibiotic Azithromycin (Figure 1).

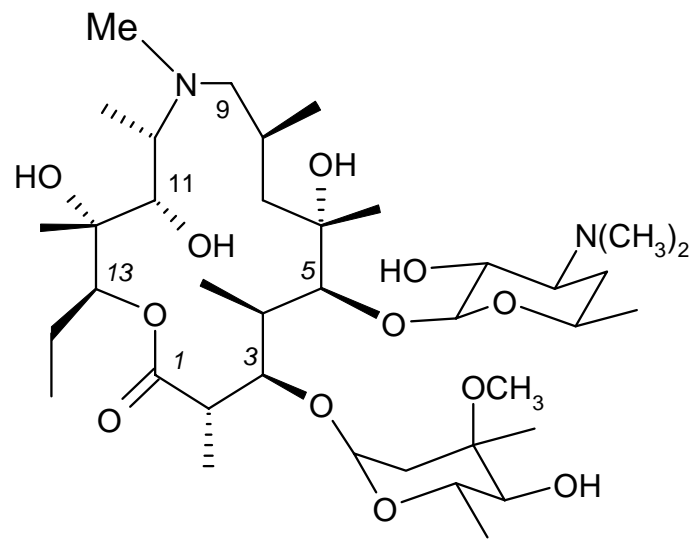

Figure 1. Azithromycin.

Azithromycin is a semi-synthetic macrolide obtained from erythromycin $\mathrm{A},{ }^{6,7}$ and is the first and most important member of a new class of antibiotics, known as azalides. ${ }^{8}$ It is an effective therapeutic agent in oral treatment for infectious diseases, showing very high antibacterial capacity (bacteriostatic) against both Gram-positive and Gram-negative bacteria. Its mode of action is preventing growth of bacteria by inhibiting protein synthesis on the ribosome, at the translocation stage.

In the solid state, Azithromycin shows at least three crystalline forms, anhydrous, ${ }^{9}$ the monohydrate (and different pseudopolymorphs hosting several solvents, mainly alcohols), ${ }^{10}$ and dihydrate, ${ }^{11}$ and four amorphous forms (containing different solvents). It is well known that the amorphous form obtained by lyophilization in $\mathrm{t}$-butanol ${ }^{12}$ transforms into the crystalline dihydrate form when placed in suspension in a mixture of water/t-butanol. ${ }^{13}$ The role of the solvent in the final crystal form is being studied. ${ }^{14}$ 
In the present work a method is developed for the detection and quantification of low amounts of the dihydrate crystalline form in presence of its amorphous counterpart.

The requirements for the dihydrate contents are less than $2 \%$. Our method is based on the increase of the intensity (measured as the area) of the highest peak in the X-ray powder diffraction pattern for the mixtures of amorphous crystal when the amount of the crystalline phase is varied in the range $0-10 \%$ (see Figure 2). A very good linear relationship was found between the percentage of crystalline phase and the area of the peak.

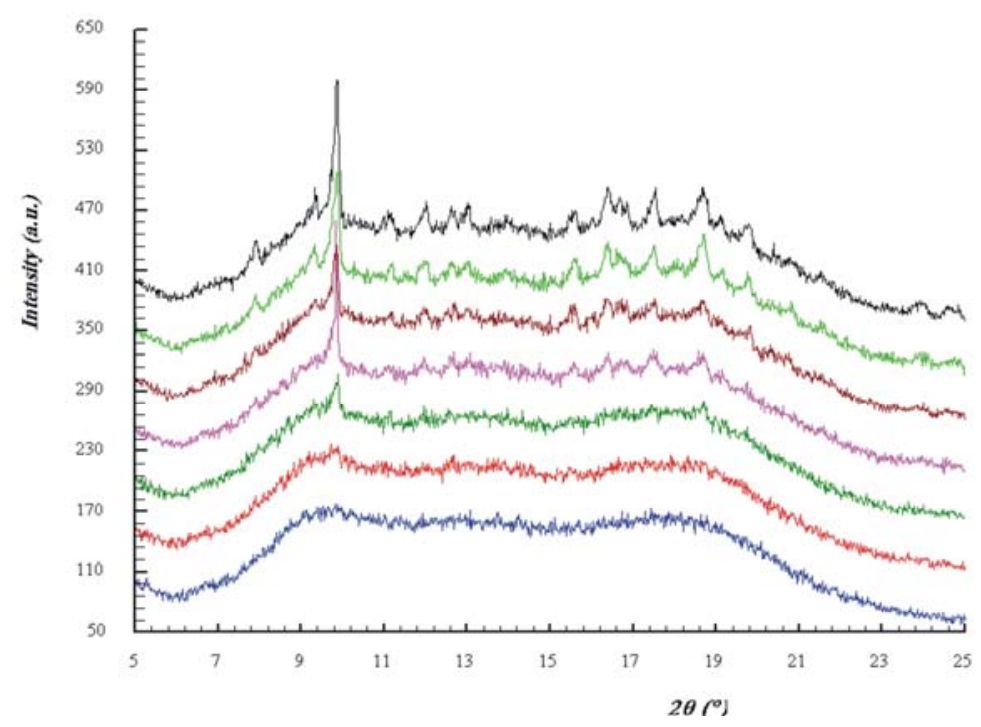

Figure 2. Powder diffraction patterns of the mixtures of amorphous and crystalline dihydrate Azithromycin (in the amounts $0 \%, 1 \%, 2 \%, 4 \%, 6 \%, 8 \%$ and $10 \%$ of the dihydrate form).

\section{Results and Discussion}

Five samples of amorphous pure Azithromycin were used. The samples, respectively labeled, azt77, le06, le07, le08 and le11, contain different amounts of water and t-butanol $\mathrm{H}_{2} \mathrm{O}: 1-1.5 \%$; t-BuOH: 3.5-5.5\%), but this feature does not have an appreciable influence on the powder diffraction pattern (see Figure 3). 


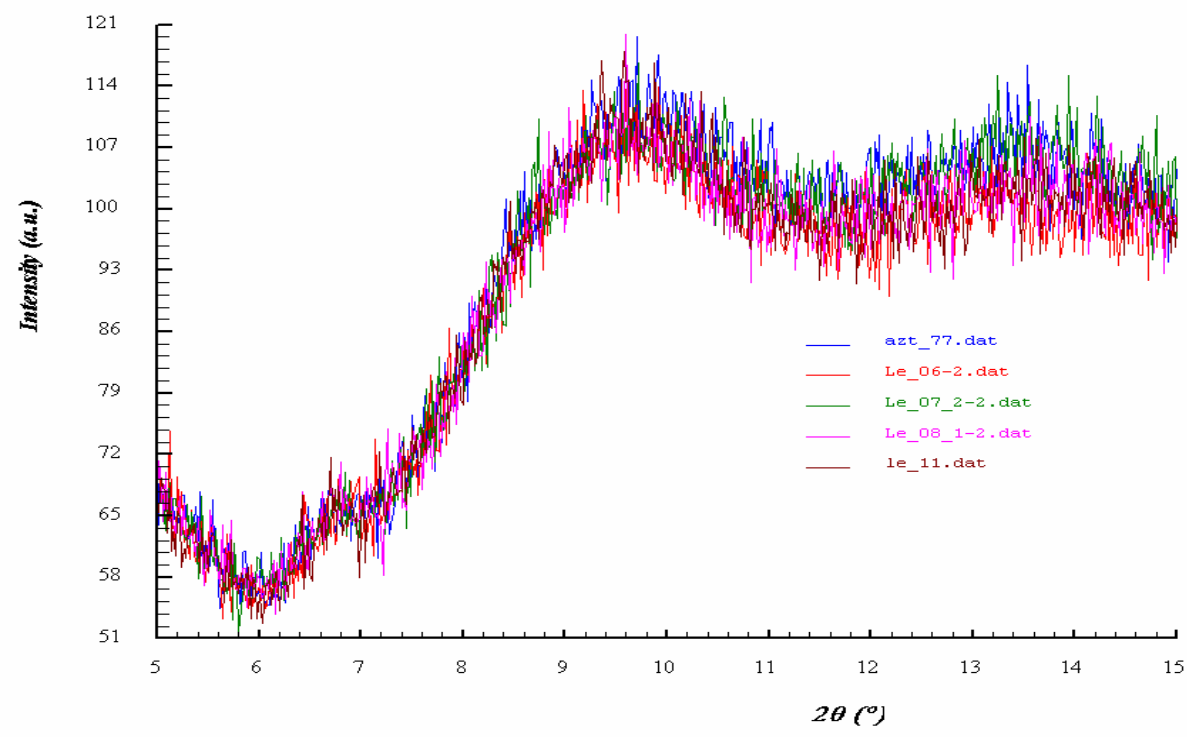

Figure 3. Powder diffraction patterns of the five amorphous samples used.3.

To obtain the mixtures of the two phases, the crystalline dihydrate phase was crushed in an agate mortar for ten minutes to obtain microcrystals with adequate size. This powder was mixed manually with the amorphous phase in several proportions $(2: 98 ; 4: 96 ; 6: 94 ; 8: 92$ and 10:90 in weight) until homogeneity, to obtain the different mixtures.

Fifty-six samples were analyzed in this study, distributed in four groups as follows.

Group 1: The five pure amorphous samples were used to obtain the point corresponding to $0.0 \%$ of crystalline dihydrate in the mixture.

Groups 2-4: Each group comprises seventeen samples, mixtures respectively of the amorphous samples le06, le07 and le08 with different percentages of crystalline Azithromycin dihydrate. Every group contains three samples with $2 \%$, three samples with $4 \%$, three samples with $6 \%$, three samples with $8 \%$, three samples with $10 \%$, one sample with $3 \%$, and one sample with $7 \%$ (the percentages indicate the amount of dihydrate form in the mixture).

The samples with percentages of $2 \%, 4 \%, 6 \%, 8 \%$ and $10 \%$ were used to obtain the experimental points for the least-squares regression, and the samples with $3 \%$ and $7 \%$ were used to validate of the linear fit obtained.

\section{Description of the method}

Our goal is to find an experimental parameter which is easily measured and is correlated with the amount of crystalline Azithromycin dihydrate in the sample (considering the sample as a mixture of crystalline and amorphous phases). The X-ray powder diffraction pattern for the mixtures of amorphous- and crystalline (Figure 2) suggests that the area of one/some peak/s could be the suitable parameter. 
In observing the diffraction pattern of the crystalline Azithromycin dihydrate (Figure 4) it becomes clear that only the highest peaks are to be considered at low concentrations. Therefore, only the peak located at $2 \Theta \approx 9.90^{\circ}$ (hereafter called the, main peak) is feasible for our purpose, and its area the experimental parameter to be measured.

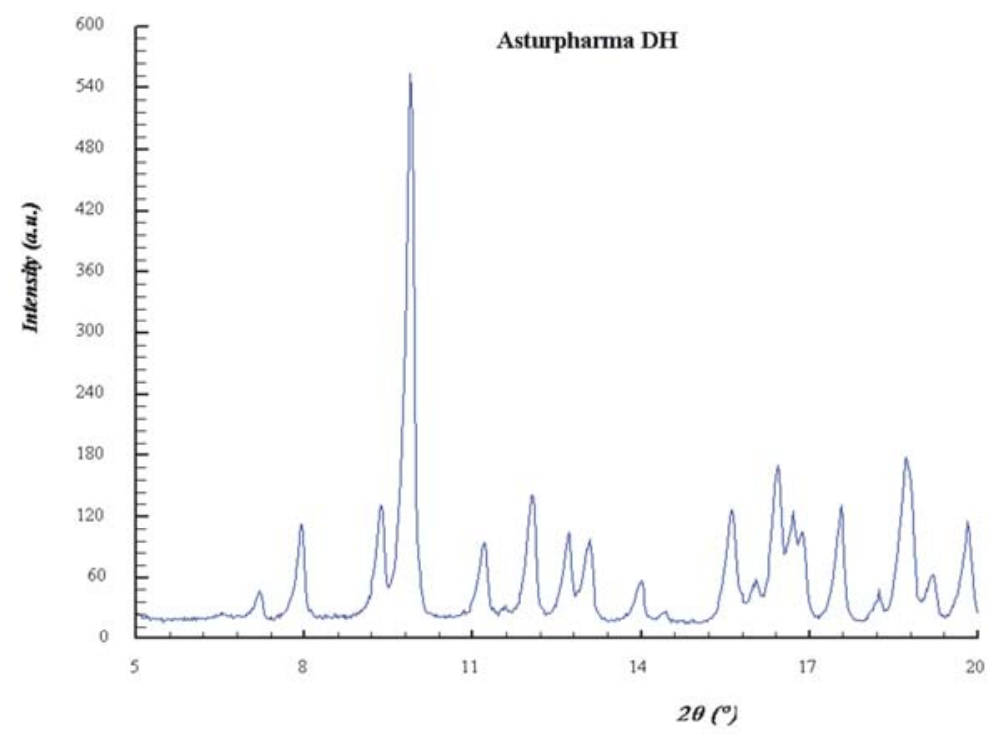

Figure 4. X-ray powder diffraction pattern of the Azithromycin dihydrate.

The analysis of the profiles obtained in the present study, (and in several previous studies considered) shows the range of $2 \Theta=9.58-9.98^{\circ}$ to be the most appropriate range of $2 \Theta$. Using these limits the tail of the main peak (for both sides) fits to the base (background) of the pattern. The background assigned to the peak is considered in the range $2 \Theta=10.10-10.50^{\circ}$ for the following reasons:

i) it is near the peak region, and therefore there are not appreciable variations due to dependences with the $2 \Theta$ angle;

ii) it does not belong to the crystalline peak profile;

iii) in this zone there is no other peak of the dihydrate pattern.

The area of the main peak is calculated by subtracting the area under the background interval to the area under the peak range (Figure 5). Finally, the area of this peak is versus the percentage of Azithromycin dihydrate present in the mixture to obtain a linear correlation between the two quantities (see Figure 6). 

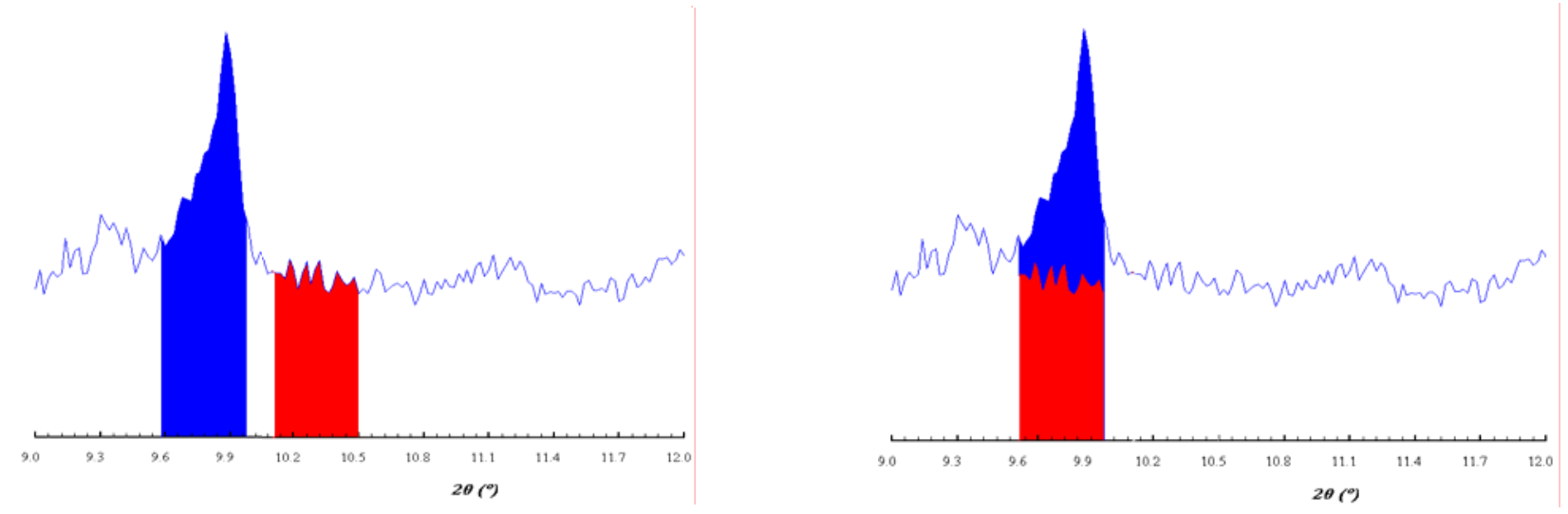

Figure 5. Calculation of the area of the main peak by subtracting the area under the background region (in red) from the area under the peak region (in blue).

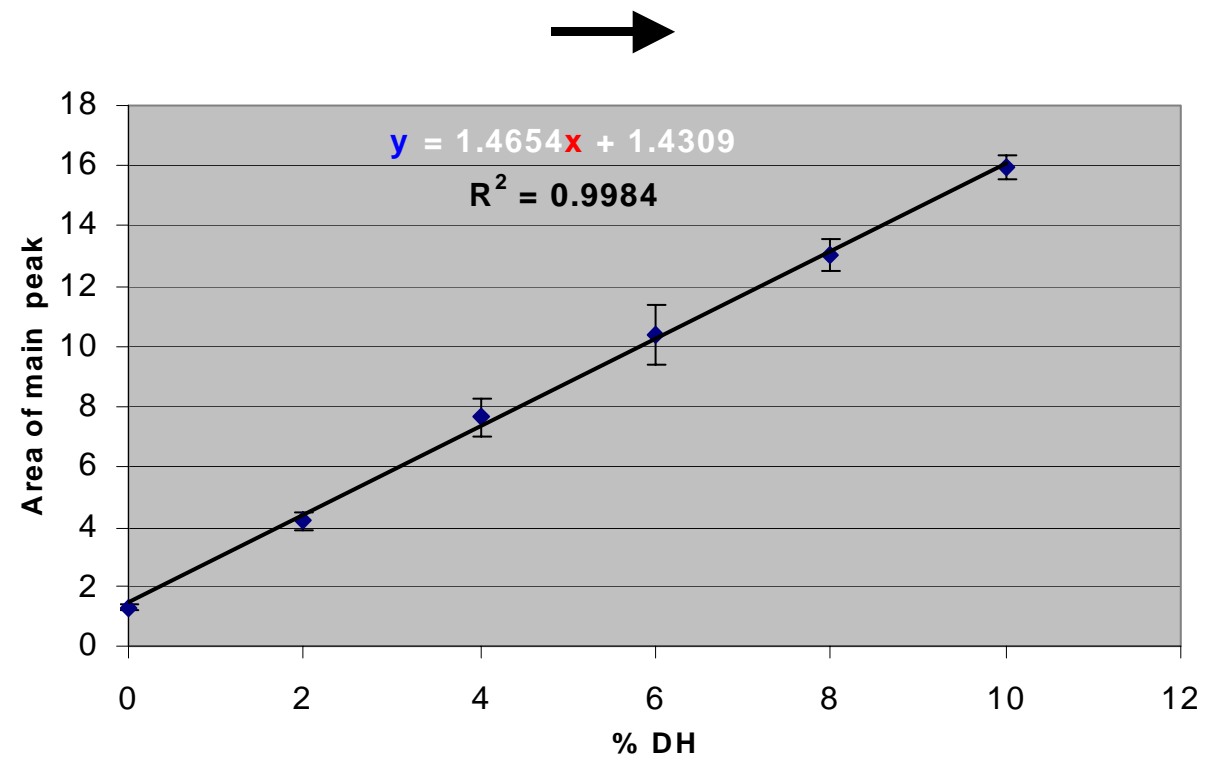

Figure 6. Plot of the main peak area as a function of the weight percentage of Azithromycin dihydrate in mixtures of amorphous and crystalline Azithromycin.

\section{Linear correlation}

For the mixture $\% \mathrm{DH}=0.0$, the values obtained by subtracting the area under the background from the area under the peak range are not zero, as might have been supposed. This results from the existence of small differences in the height of the profile for both intervals. The final area obtained for the five samples of pure amorphous material were (in units of area): 1.25 (azt77), 1.23 (le06), 1.27 (le07), 1.72 (le08) and 1.40 (le11). To calculate the mean value, the value for le08 was rejected for being very different from the rest. 
Nine values were measured for the remaining concentrations of crystalline dihydrate in the mixtures, but only seven were considered: for each mixture the extreme results were rejected. For the $8 \%$ case, however, only three experimental values were considered because the rest were higher than for the $10 \%$ case. The experimental results used to fit the straight line are collected in Table 1 and the fitting graph is shown in Figure 5.

Table 1. Values of the area of the main peak for the different mixtures

\begin{tabular}{rrrrrrrrrr}
\hline$\% \mathrm{DH}$ & \multicolumn{1}{c}{ Value of the area of the mean peak } & & & Mean & e.s.d. \\
\hline $\mathbf{0 . 0 0}$ & 1.23 & 1.25 & 1.27 & 1.40 & & & & 1.29 & 0.08 \\
$\mathbf{2 . 0 0}$ & 4.03 & 4.27 & 4.65 & 4.10 & 4.25 & 4.46 & 3.71 & 4.21 & 0.30 \\
$\mathbf{4 . 0 0}$ & 8.00 & 7.00 & 7.48 & 7.56 & 6.80 & 8.30 & 8.49 & 7.66 & 0.64 \\
$\mathbf{6 . 0 0}$ & 9.94 & 11.09 & 9.85 & 9.40 & 11.64 & 9.40 & 11.50 & 10.40 & 0.98 \\
$\mathbf{8 . 0 0}$ & 12.58 & 13.60 & 12.85 & & & & & 13.01 & 0.53 \\
$\mathbf{1 0 . 0 0}$ & 15.50 & 16.50 & 15.85 & 16.18 & 16.25 & 16.10 & 15.44 & 15.97 & 0.40 \\
\hline
\end{tabular}

The percentage of Azithromycin dihydrate in a given sample of amorphous Azithromycin (x) is calculated simply as, $\mathbf{x}=(\mathbf{y}-1.4309) / 1.4654$, where $\mathbf{y}$ is the experimental area of the main peak. Several test examples will be shown.

\section{Detection and quantification}

In any quantification method it is very important to know its limit of detection and quantification. The first can be defined as, "the minimum analyte concentration that can be detected for a determined level of confidence $(>90 \%)$ and/or gives a signal in the instrument significantly different that the given by the blank" and can be calculated using two different equations (giving different results):

$$
\begin{aligned}
& \mathbf{X}_{\text {det } \lim }=\mathbf{3} \cdot \mathbf{S}_{\mathbf{b} \mathbf{1}} / \mathbf{m} \quad \text { Eqn. } 1 .^{15} \\
& \mathbf{X}_{\text {det } \lim }=\mathbf{3} \cdot \mathbf{S}_{\mathbf{y} / \mathbf{x}} / \mathbf{m} \quad \text { Eqn. } \text { 2. }^{16}
\end{aligned}
$$

where $\mathbf{S}_{\mathbf{b l}}$ is the standard deviation of the blank signal (considered here as the case $\% \mathrm{DH}=0.0$ ), $\mathbf{S y} / \mathbf{x}$ is also the standard deviation of the blank signal, but considering residual values $\left(\mathrm{y}_{\mathrm{I}}-\mathrm{y}_{\mathrm{i}}{ }^{*}\right)$, where $\mathrm{y}_{\mathrm{i}}{ }^{*}$ are the values for $\mathrm{x}_{\mathrm{i}}$ calculated with the fitting straight line), and $\mathbf{m}$ is the slope of the straight line. In our case, for the Azithromycin dihydrate, the limit of detection fitting according to the two equations is:

$$
\begin{aligned}
& \mathbf{X}_{\text {det } \lim }=3 \cdot 0.077 / 1.4654=0.16 \% \mathrm{w} / \mathrm{w} . \\
& \mathbf{X}_{\text {det } \lim }=3 \cdot 0.248 / 1.4654=0.50 \% \mathrm{w} / \mathrm{w} .
\end{aligned}
$$

The limit of quantitation, defined as, "the minimum amount that can be quantified" is generally taken as ten times the standard deviation of the blank signal $(\% \mathrm{DH}=0.0)$. In our case, for the Azithromycin dihydrate, the limit of quantification is:

$$
\mathbf{X}_{\text {quan lim }}=\mathbf{1 0 S}_{\mathrm{bl}} \Rightarrow 10 \cdot 0.077=0.77 \% \mathrm{w} / \mathrm{w} \text {. }
$$




\section{Secondary correlation}

A linear correlation between the percentage of Azithromycin dihydrate in the mixture and the area under a specific region of the pattern was found. The fitting is worse that in the previous correlation, and we call this linear relationship a secondary correlation.

The principle is that there is a relationship between the percentage of Azithromycin dihydrate in the mixture, and the area of the region under the experimental profile in the interval $\mathbf{2 \theta}=$ $10.0-15.0^{\circ}$ (hereafter, this area will be called the reference area). In Figure 7 is shown the relationship obtained for the 15 mixtures using the pure amorphous sample le07. For the mixtures using the pure amorphous samples le06 and le08 the value of goodness of fit were respectively, $\mathrm{R}^{2}=0.8052$, and $\mathrm{R}^{2}=0.9325$.

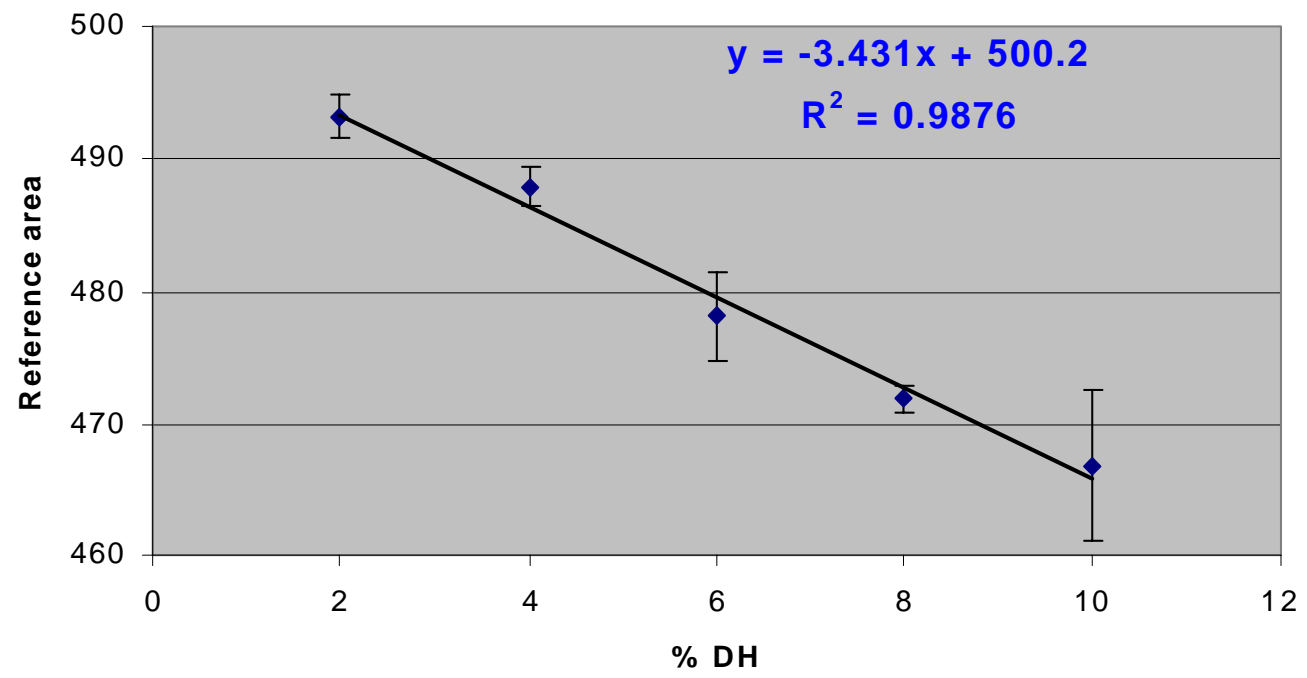

Figure 7. Plot of the Reference area as a function of the weight percentage of Azithromycin dihydrate in mixtures with the pure amorphous sample le07.

Since this correlation is not very good, it cannot be used to calculate the amount of Azithromycin dihydrate in an experimental mixture, but it can indicate the presence of the crystalline phase (values of the reference area below 500) and gives a first estimate of the amount (according to the values of the reference area).

\section{Scale of the experimental patterns}

The fact that the intensity of the patterns decreases with time is an additional problem since, for the same concentration of dihydrate, profiles measured at different times will give different values for the area of the main peak, and therefore given us different values of percentages for the dihydrate. 
In order to try to correct these results, the experimental profiles have to be multiplied by a scale parameter (SP) to put them on the same scale of intensities as is used for determining the calibration curve. This scale parameter is calculated comparing: (a) the value of the reference area of a pure amorphous sample measured at the same time as the samples we want to analyze, with: (b), the mean value of the reference area for the five samples of pure amorphous material used to calculate the point $[\mathrm{DH}]=0.0$. This mean value is 509.31 , and this will always be bigger than the value obtained in (a). To obtain the scale parameter it is only necessary to divide 509.31 by the value of (a). We shall show the validity of this method later on, with some examples.

\section{Experimental validation of the method}

Several experimental results have proved the validity of the method demonstrated here.

(i) The three mixtures with $3.00 \%$ and $7.00 \%$ of dihydrate, made to validate the fitting to a straight line, gave mean values of $3.29 \%$ and $6.88 \%$ respectively!

(ii) Four more samples measured recently (and the corresponding pure amorphous material, to calculate the scale parameter), show the mean value of the reference area to be 268.48 , while the value for the pure amorphous phase was 267.10. With these results, the corresponding scaling parameter was $1.91(509.31 / 267.10)$, and the concentration of dihydrate expected was $\approx 0.0$ (since the value of the reference area for the four samples investigated is very similar to that of the pure amorphous sample). In fact, after scaling the patterns, the percentages of dihydrate obtained were very small: $0.66 \%, 0.45 \%, 0.79 \%$ and $0.62 \%$.

(iii) A sample with the clear presence of dihydrate component (the experimental pattern shows clearly the main peak) and multiplied by a scale parameter of 2.25 (obtained from the measure of the reference area of the pure amorphous sample measured at the same time) showed an amount of dihydrate phase of $3.11 \%$.

The same sample was also analyzed using a method based in the use of the NIR technique, ${ }^{17}$ as the reference method, (the NIR technique has been used in recent years for this kind of study) ${ }^{18}$ and the obtained result, $2.90 \%$, compares very well with that obtained using our method.

\section{Conclusions}

An easy and reliable quantification method has been developed to quantify crystalline Azithromycin in an amorphous matrix. This is based on the X-ray diffraction intensity of the main peak of the crystalline phase. The procedure can easily be applied to mixtures containing less than $10 \%$ of crystalline phase.

The expressions for the calibration curves are given. The calibration curves are linear with a confidence level, R2 > 95\%. The limits of detection and quantification achieved with our method are $0.50 \% \mathrm{w} / \mathrm{w}$ and $0.77 \% \mathrm{w} / \mathrm{w}$ respectively. 
All tests performed with real mixtures have been highly satisfactory, and the procedure is still valid for scale parameters higher than 2.00. The procedure is likely to be applied for quantification of minor crystalline phases in many other amorphous samples.

\section{Experimental Section}

X-Ray General Procedures. X-ray powder diffraction patterns for the 56 samples were collected at room temperature on the X-ray diffractometer (Seifert XDR $3000 \mathrm{~T} / \mathrm{T}$ ), using $\mathrm{CuK}_{\alpha}$ radiation (tube operated at $50 \mathrm{kV}, 40 \mathrm{~mA}$ ). Data were collected in the step-scan mode, over a $2 \Theta$ angular range from $5^{\circ}$ to $15^{\circ}$, using a step size of $0.02^{\circ}$ and a step time of 10 secs. The pattern profile plots were made with the WinPLOTR (FULLPROF ${ }^{19}$ program package).

\section{General procedure for obtaining pure amorphous Azithromycin ${ }^{12}$}

Crystalline Azithromycin monohydrate $(100 \mathrm{~g})$ is dissolved in tert-butanol $(500 \mathrm{ml})$ heated at $30^{\circ} \mathrm{C}$. The solution is filtered, and solidified in a cooling bath. The solvent is sublimed at room temperature and $10^{-2} \mathrm{~mm} \mathrm{Hg}$. The solid obtained is dried $\left(80 \mathrm{~mm} \mathrm{Hg} / 40^{\circ} \mathrm{C}\right)$ to yield $100 \mathrm{~g}$ of non-crystalline Azithromycin.

\section{General procedure for obtaining Azithromycin dihydrate (crystalline) ${ }^{12}$}

Crystalline Azithromycin monohydrate $(25 \mathrm{~g})$ is dissolved in tert-butanol $(130 \mathrm{ml})$ heated at $30^{\circ} \mathrm{C}$. This solution is filtered, then water $(130 \mathrm{ml})$ added over $6 \mathrm{~h}$. The resulting mixture is taken to $\mathrm{pH}=11$ by addition of $2 \mathrm{~N} \mathrm{NaOH}$, cooled to below $10^{\circ} \mathrm{C}$ and then stirred for $48-72 \mathrm{~h}$. The crystals are collected by filtration and dried $\left(80 \mathrm{mmHg} / 25^{\circ} \mathrm{C}\right)$ to yield $15 \mathrm{~g}$ of Azithromycin dihydrate.

\section{Acknowledgements}

The authors thank the Spanish MCyT (BQU2003-05093), Principado de Asturias FICYT (PR01-GE-04) and AsturPharma S.A. (FUO-EM-132-02) for financial support.

\section{References}

1. Chen, X.; Bates, S.; Morris, K.R. J. Pharm. Biomed. Anal. 2001, 26, 63.

2. Fix, I.; Steffens, K.J. Drug Devel. Ind. Pharm. 2004, 30, 513.

3. De Villiers, M.M.; Wuster, D.E.; Van der Watt, J.G.; Ketkar, A. Int. J. Pharm. 1998, 163, 219.

4. Orlhac, X.; Fillet, C.; Deniard, P.; Dulac, A.M.; Brec, R. J. Appl. Cryst. 2001, 34, 114. 
5. Surana, R.; Suryanarayanan, R. Powder Diffr. 2000, 15, 2.

6. Djokic, S.; Kobrehel, G. U.S. Patent 1985, US 4517359.

7. Bright, G.M. U.S. Patent 1984, US 4474768.

8. Bright, G.M.; Nagel, A.A.; Bordner, J.; Desai, K.A.; Dibrino, J.N.; Nowakowska, J.; Vincent, L.; Watrous, R.M.; Sciavolino, F.C.; English, A.R. J. Antibiot. 1988, XLI, 1029.

9. De la Torre-García, J.A.; Franco-Andrade, F.; Lara-Ochoa, J.M.F. Int. Patent 2002, WO $02 / 10144$.

10. Montejo-Bernardo, J.M.; Garcia-Granda, S; Bayod-Jasanada, M.S.; Llavona-Díaz, L.; Llorente, I. Z. Kristallogr. 2003, 218, 1.

11. Djokic, S.; Kobrehel, G.; Lopotar, N.; Kamenar, B.; Nagl, A.; Mrvos, D. J. Chem. Res. (M) 1988, 1239; J. Chem. Res. (S) 1988, 152.

12. Bayod-Jasanada, M.; Llorente-García, I.; Fernandez-Mari, F. Eur. Pat. Appl. 2001, EP 1103558.

13. Bayod, M. Personal communication.

14. Montejo-Bernardo, J.M.; García-Granda, S; Bayod-Jasanada, M.S.; Llavona-Diaz, L.; Llorente, I. Z. Kristallogr. 2005, 220, 66.

15. Skoog, D.A.; Leary, J.J. In Instrumental Analysis, McGraw-Hill 1994; $4^{\text {th }}$ Edn, Ch. 1.

16. Miller, J.C.; Miller, J.N. In Statistics for Analytical Chemistry, Addison-Wesley Iberoamericana 1993; $2^{\text {nd }}$ Edn, Ch. 5.

17. Blanco, M.; Valdes, D.; Bayod, M.S.; Fernandez-Mari, F.; Llorente, I. Anal. Chim. Acta. 2004, 502, 221.

18. Gombas, A.; Antal, I.; Szabo-Revesz, P.; Marton, S.; Eros, I. Int. J. Pharm. 2003, $256,25$.

19. "FULLPROF: a program for Rietveld refinement and pattern matching analysis." J. Rodriguez-Carvajal. Abstracts of the Satellite meeting on Powder Diffraction of the XV Congress of the IUCr, p. 127. Toulouse, France. 1990. 